\title{
Predictive performance estimation for a dual-battery system in mild-hybrid vehicles
}

\author{
Daniel Renner ${ }^{1}$, Patrick Jansen ${ }^{1}$, David Vergossen ${ }^{1}$, Werner John ${ }^{2}$, and Stephan Frei ${ }^{3}$ \\ ${ }^{1}$ Audi Electronics Venture GmbH, Gaimersheim, Germany \\ ${ }^{2} \mathrm{SiL}$ GmbH, Paderborn, Germany \\ ${ }^{3}$ TU Dortmund University, AG On-Board Systems, Dortmund, Germany \\ Correspondence to: Daniel Renner (daniel1.renner@audi.de)
}

Received: 15 January 2016 - Revised: 9 May 2016 - Accepted: 25 May 2016 - Published: 28 September 2016

\begin{abstract}
Continuously increasing requirements for onboard system performances lead to new topologies for the energy distribution in vehicles. One promising concept is the usage of a dual-battery system instead of the conventional lead-acid "starting lightening ignition" battery. As this system is not able to control the current share between the two batteries, its performance depends on the actual battery specific operating points. The initial conditions of state of charge, voltage level and temperature influence the current share and lead to a different voltage drop of the system. This paper yields to, the basic understanding of the current share between the two batteries. The conventional performance estimation method for standalone lead-acid batteries can no longer be applied to this system. Therefore, a new algorithm for the voltage drop calculation of the dual-battery system is proposed. Measurements at different temperatures, states of charge and voltage levels show the system behavior and prove the functionality of the algorithm.
\end{abstract}

\section{Introduction}

The energy supply of today's vehicles is getting increasingly attention due to fuel economy and autonomous driving functions (Gehring et al., 2009). Given that more and more parts in modern vehicles are electrified for fuel economy reasons, the need for a steady, high-performance power supply is gaining importance. Additionally, autonomous driving functions such as piloted driving call for a fail-safe concept for the electrical energy supply. Furthermore, new driving functions such as "start/stop" and "engine-off coasting", or highperformance consumers as electrical superchargers require a very powerful battery system.Besides these requirements, being able to determine and predict which performance the battery achieves, is equally important for a good customer experience and overall reliability.

State-of-the-art-systems used in conventional vehicles are standalone lead-acid batteries (LAB). This battery type has a very limited ability to serve these new functions due to its poor charge acceptance. Additionally its low voltage level coupled with a high internal resistance leads to a significant voltage drop and possible malfunction.

Thus, new topologies for the energy supply system of conventional vehicles are required. One promising topology is the dual battery system (DBS) published in articles of Schindler (2014) and Zhang et al. (2015). The larger dimensioned $\mathrm{LAB}$ provides cranking power and the energy during the ignition-off phases, while the smaller lithium-ion battery (LIB) is used for charge acceptance and stabilizing the power system. Besides the cell type and chemistry of the two batteries, their capacity and connection to the power network are essential factors in developing the system (Rizoug et al., 2014). The combination of two batteries reduces the combined internal resistance for high performance applications on the one hand and brings the redundancy needed for autonomous driving functions on the other hand. Using these two batteries at the $12 \mathrm{~V}$ voltage level means that no additional components such as a relay or a power converter are necessary in this system, which reduces complexity and costs. At the same time, the power distribution and the current share between the two batteries cannot be controlled. Given that the current share depends on the battery's operating points, it differs with different states of charge (SOC), voltage levels or temperatures. For power estimation pur- 


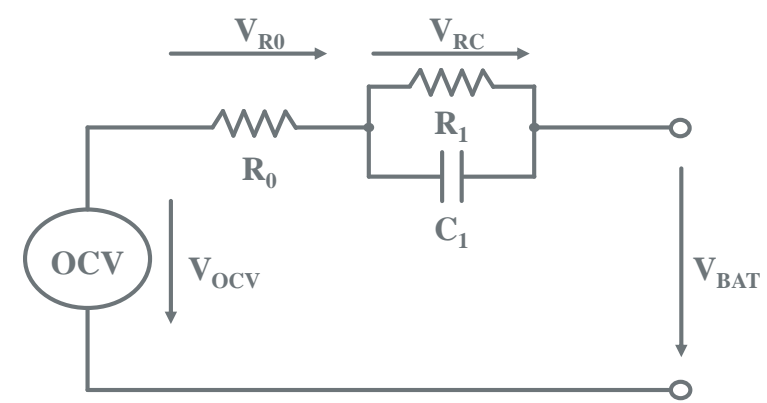

Figure 1. Equivalent circuit diagram of a battery, also called Thevenin Model

poses the voltage drop at a defined load current is calculated. For standalone LABs the voltage drop is calculated by multiplying the internal resistance to the predicted load current. Given that the batteries in use have different cell chemistries as well as open circuit voltage (OCV) levels, this approach is not expedient for the DBS. A precise estimation of the DBS performance in combination with a powerful DBS can extend features such as "start/stop" or "coasting". Furthermore, this combination ensures a stable power network and thereby increases the availability of high performance applications.

This paper describes a new method taking into consideration the batteries' different internal resistances and OCV levels. It starts with an overview of different possibilities to model a battery for our purpose. The most suitable model for this application is used to illustrate the current share between the two batteries at different operating points. In the third section, the three different performance estimation procedures used for the proposed algorithm are presented. Then, the combination of the presented procedures is described. The results of the predictive estimation compared with vehicle measurements at different voltage- and SOC-levels are shown in the last section and prove the effectiveness of the proposed method.

\section{Methods for battery modelling}

Battery models can be classified in different detail levels depending on demands of computing efficiency and accuracy. Models with the best accuracy are those considering the physio-chemical process during charging or discharging (Thele et al., 2007). This type of models, are very detailed and are valid for various time constants and power ranges. However, they have a very high computational complexity, need expert knowledge for parametrization and are valid for only one specific type of battery. The second type of models, the empirical mathematical models use mathematical abstractions for the complex processes in a battery. They have a great computational efficiency, at a moderate accuracy and are working mostly just in one operating point.

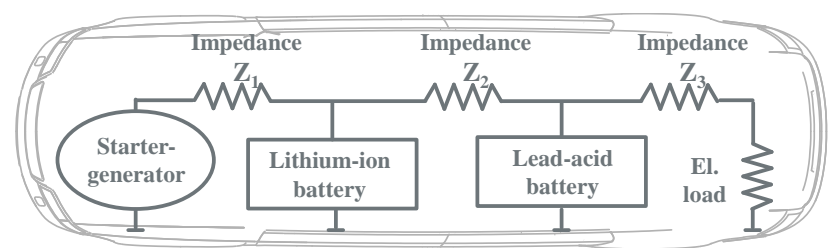

Figure 2. Vehicle power network with dual-battery system

\subsection{Equivalent circuit battery models}

An equivalent circuit model is another way to model batteries. This model type offers the best trade-off between accuracy and computational complexity. Numerous different equivalent circuit models, also varying in complexity, can be found in literature (Chen and Rincon-Mora, 2006). Figure 1 shows the Thevenin-Model approach. It consists of an OCV source $V_{\mathrm{OCV}}$ showing the voltage of the battery while no charge is applied. The resistance $R_{0}$ represents the battery's ohmic resistance, which represents the voltage drop inside the battery caused by terminals and arresters. The $R_{1}$ $C_{1}$-circuit represents several different, time-based, chemical reactions, such as diffusion or the charge carrier movement.

Having only one RC circuit, this approach for an equivalent circuit model is only possible to represent processes in the same time constant range. The model is used here just in discharge direction and for time constants up to a onedigit number of seconds, thus it fits for our approach. According to Netter et al. (2006) one RC branch model offers the best trade-off between computational efficiency and accuracy. The main disadvantage of the Thevenin battery model is that all the elements are assumed to be constant. In fact, all the values are functions of battery conditions. Thus, we need to consider the variations in parameters accompanied with different battery conditions for precise power estimations, since a battery's performance depends on the SOC, temperature and current density, disregarding ageing. Thus, we expand the Thevenin-Model with dependencies for every component as follows:

$V_{\mathrm{OCV}}=f(\mathrm{SOC})$

and

$R_{0}, R_{1}, C_{1}=f(\mathrm{SOC}$, Temperature, Current)

\subsection{Dual-battery storage in a parallel connection}

The topology shown in Fig. 2 is the result of connecting the batteries in parallel through the vehicle's wiring harness. Section 2.3 describes the LAB and LIB used for DBS. Various locations for the batteries are possible, but we concentrate on a parallel connection next to each other at the vehicle's trunk.

For estimation of the voltage drop at the $\mathrm{LAB}$, the impedance of the wire $Z_{2}$ used to connect the batteries in 


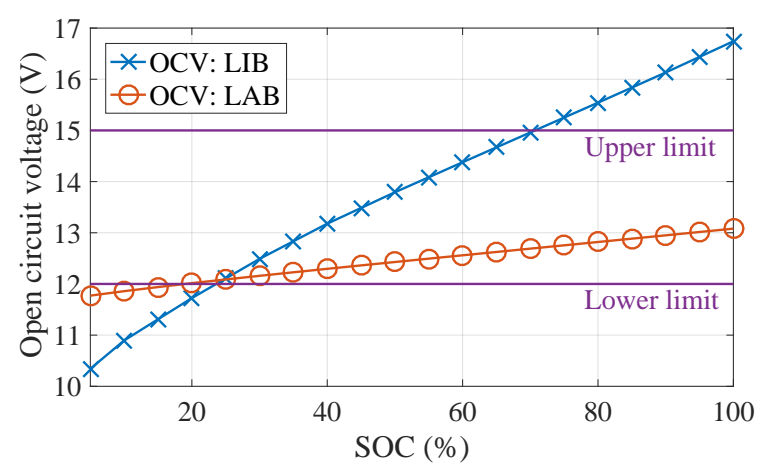

Figure 3. Open Circuit Voltages of LIB and LAB

parallel, has to be considered as well. In this case the internal resistance of the LIB $R_{0}$ LIB has to be extended by

$R_{0 \mathrm{LIB}}^{\prime}=R_{0 \mathrm{LIB}}+Z_{2}$.

\subsection{Batteries used in the test setup}

Given that the two batteries are connected in parallel, the behavior of one battery directly influences the other battery's performance. Therefore, the parameters of the batteries in use are shown in the same diagram for a better comparison. The LAB used for the test setup has an Absorbed Glass Mat (AGM) design with a capacity of $70 \mathrm{Ah}$. In this design the $\mathrm{H}_{2} \mathrm{SO}_{4}$ ) electrolyte is held in the glass mat, as opposed to freely flooding the plates. Very thin glass fibers are woven into a mat to increase the surface area enough to hold sufficient electrolyte on the cells for their lifetime. This reduces coating and ensures an improved lifetime. In the DBS the LAB is used for cranking and during the ignition-off periods as it is able to accumulate a huge amount of energy. The battery used for charge acceptance and stabilizing the power system is a LIB with $10 \mathrm{Ah}$ and a cathode material of a Nickel, Manganese, Cobalt (NMC) blend. The anode material is carbon. Being designed for power applications, the cells have a very high cyclical lifetime even though treated with high currents.

Figure 3 shows the OCV curve of the two batteries in use at different SOC levels. The two horizontal lines show the typical maximum and minimum voltages occurring in a vehicle's operation. During recuperation periods, the alternator voltage rises up to the maximum level to recharge the DBS using the kinetic energy of the vehicle. In contrast, at engineoff periods the power network voltage may drop to a level close to the minimum limit.

Most of the LAB's OCV curve is located between the typical voltage limits, while the LIB covers the whole LAB range and can only be used in a part of its SOC band. A LIB operation at low SOC levels in combination within a small SOC window achieves high cycle stability and long lifetime. To get a steady state with a parallel connection, the voltage levels of the two batteries have to be identical. Possible steady

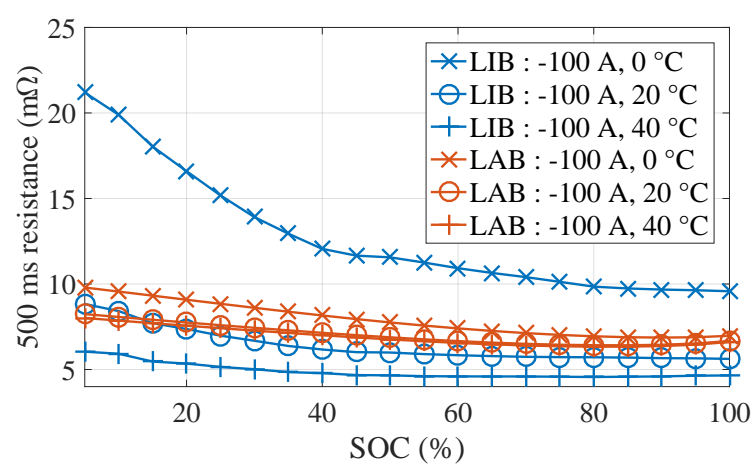

Figure 4. Discharge resistances after a $500 \mathrm{~ms}$ and $100 \mathrm{~A}$ discharge pulse at different temperatures

states would be for example at voltage levels of $12.2 \mathrm{~V}$ with a $\mathrm{SOC}_{\mathrm{LIB}}$ of $26 \%$ and a $\mathrm{SOC}_{\mathrm{LAB}}$ of $33 \%$ or at a voltage level of $12.8 \mathrm{~V}$ with a fully charged LAB and a LIB with a SOC of $37 \%$. But since the LAB has some special characteristics it is possible to get steady states above the LAB's OCV curve. Firstly, there is a phenomenon, the so-called overvoltage, which appears at SOC levels greater than $60 \%$. During and after a charge process the voltage level on the battery terminals appears to be higher than the regular OCV level, caused by coating and other side effects. This overvoltage results in an additional voltage of up to $400 \mathrm{mV}$ (Naumann, 2004). Secondly, the parameters of a battery depend on the SOC and current density as well as current direction. While the internal resistance for discharging are shown in Fig. 4 the internal resistance at a charging procedure differ widely. During charging at high current rates or high SOC's under dynamic operation there is a depletion of $\mathrm{Pb}^{2+}$ ions. This is responsible for the limited charge acceptance and can be modeled with a high internal resistance of the LAB at these conditions. The decrease of the $\mathrm{Pb}^{2+}$ ion concentration is caused by the generation of $\mathrm{Pb}$ and $\mathrm{PbO}_{2}$ from $\mathrm{Pb}^{2+}$ ions in the negative and the positive electrode. This causes a significant overvoltage and the charge acceptance of the battery is restricted, if maximum charging voltage limits are used, as in our study (Thele et al., 2007). Due to these two attributes of the LAB the charge reversal is reduced to a minimum. So, for the time constants appearing during vehicle operation in combination with the omnipresent current oscillations in vehicle's power networkss we are able to talk of a steady state, for example at a $14 \mathrm{~V}$ level with a fully charged LAB and a LIB with an SOC of $54 \%$. As the current distribution determines the DBS performance we are interested in the internal resistances during discharge operation. Figure 4 shows the resistances at different SOC levels and three different temperatures. The resistances were identified by applying a $100 \mathrm{~A}$ pulse current for $500 \mathrm{~ms}$ length of time in discharge direction. The typical characteristic of all electrochemical storages is, that with higher temperatures the resistance decreases to a lower level, as the chemical reactions process faster. The 


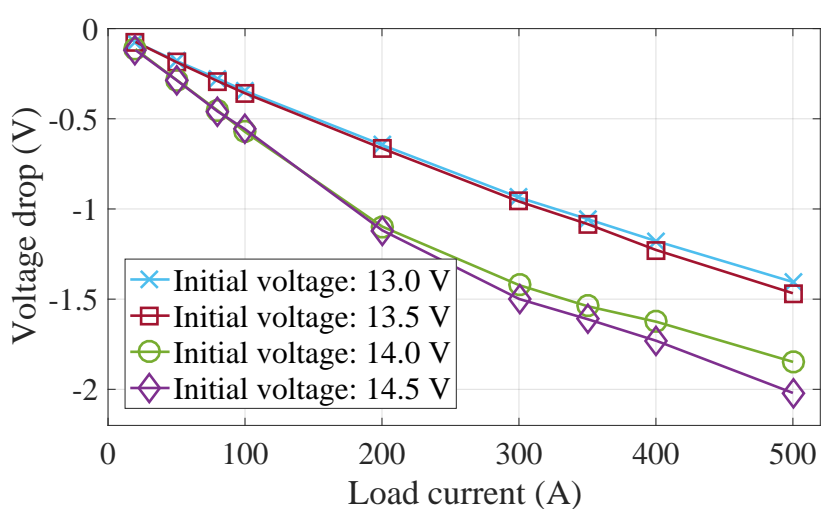

Figure 5. DBS voltage drop at different load currents and initial voltage levels

diagram shows, that the LIB reacts more sensitive to lower temperatures than the LAB.

The $R_{1}$ and $C_{1}$ are not shown for the batteries, as they do not differ much with SOC variation.

\section{Performance estimation of the Dual-Battery System}

Figure 4 shows that the internal resistances for the LIB and the LAB differ at a variation of SOC and temperature. Due to the variation of internal resistances, the current distribution between the two energy storages shifts towards the battery with a minor internal resistance. Thus, this battery has to take more load current and is the determining factor regarding the voltage drop. However there are more influencing variables as just the relationship between the resistances shown in this section.

\subsection{Voltage drop during vehicle's operation}

The voltage window for the DBS during vehicle's operation is shown in Fig. 3. Within this window the energy management of the vehicle has to be able to predict the voltage drop of any applied power load. Hence, Fig. 5 shows the voltage drop after one second on-load operation of the DBS at varied initial voltages before the applied load current.

First of all, the Butler-Volmer-Characteristic, which is typical for batteries, exists for the DBS system as well (Atkins et al., 2006). It describes how the electrical current on an electrode depends on the electrode potential, considering that both a cathodic and an anodic reaction occur on the same electrode. Simplified, it claims, that the batteries' resistance is higher when getting discharged with a minor load, and gets lower, when a major load is applied. This explains, why in Fig. 5 is no direct correlation between the voltage drop and load current. The figure shows the impact of the initial voltage before the load is applied. The test setup was made at identical battery temperatures of $25^{\circ} \mathrm{C}$. Due to a very small change in SOC, the internal resistance remains the same. Ap-

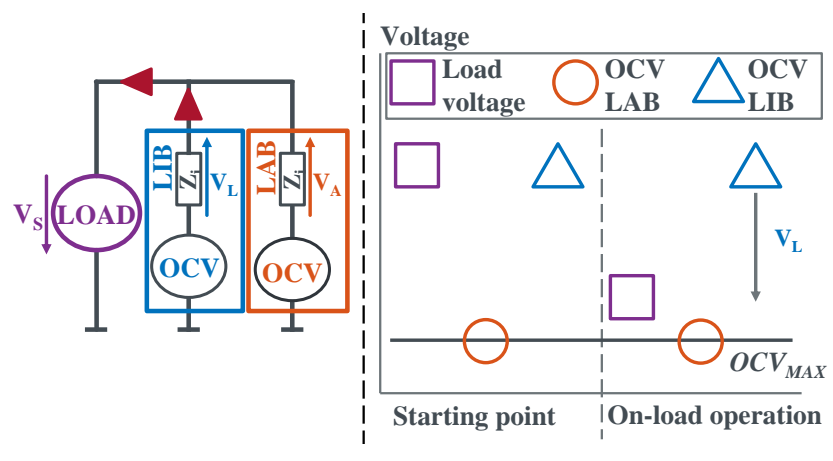

Figure 6. DBS Performance - Case A: Single battery distribution.

plying load to the DBS at voltage levels above the OCV of the LAB, another effect occurs, explained in the next section.

\subsection{Theory of current distribution in a Dual-Battery System}

Depending on the initial voltage level and the applied load power, there are three variations for the current distribution and therefore the predictive performance estimation.

\subsubsection{A - Single battery distribution}

Possibility one for the current distribution in the DBS is shown in Fig. 6.

This case takes place, if the voltage level in the power system is above the maximum OCV of the LAB, OCV MAX. The maximum OCV in this context means, the LAB's OCV at the corresponding SOC plus the overvoltage generated by pervious charging. As the LIB is charged to a voltage potential higher than the LAB OCV, it stabilizes the voltage at this level. So the vehicles' consumer loads are on the same voltage level. There is no current reversal due to overvoltage and charge acceptance of the LAB in this case. Applying a load to the DBS, as long as the load current is not as high that, the voltage drops below the OCV $\mathrm{MAX}$ level, the LAB does not get active. Therefore, the LIB takes the whole current and the performance has to be estimated as shown in equation 4 . Taking the equivalent circuit diagram in Fig. 1 as a basis, the voltage drop $V_{\text {Drop }}$ for the LIB applied with a load current $I_{S}$ is shown in the differential equation

$V_{\text {Drop }}=I_{S} \cdot\left(R_{0}+R_{1}\right)-\left(R_{1} \cdot C_{1}\right) \dot{V}_{R_{1} C_{1}}$.

To estimate the voltage drop, we solve the differential equation and convert it into the form in Eq. (5).

$V_{\text {Drop }}=Z_{i} \cdot I_{S}$

The impedance $Z_{i}$ contains the time depended parts of the Tevenin Modell and is shown in Eq. (6).

$Z_{i}=R_{0}+R_{1} \cdot\left(1-\exp \left(-\frac{1}{R_{1} \cdot C_{1}}\right) \cdot t\right)$ 


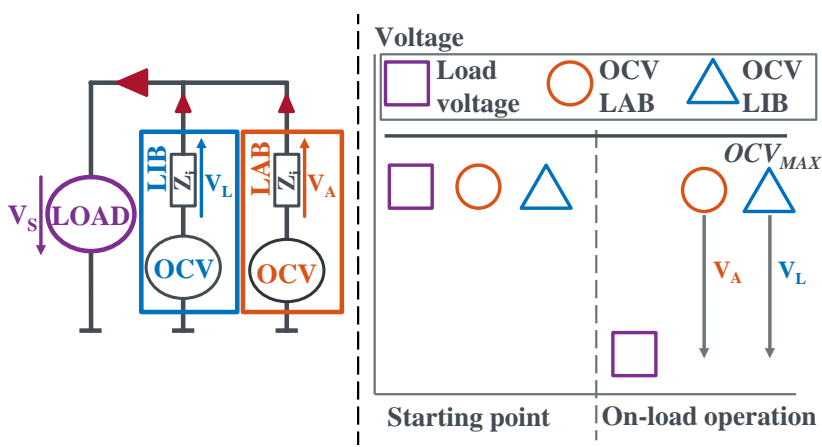

Figure 7. DBS Performance - Case B: Split current distribution.

To estimate the resulting "On-Load Operation voltage" $V_{\mathrm{OLO}}$ during the applied load Eq. (7) is suitable. Equation 7 calculates $V_{\mathrm{OLO}}$ taking the voltage at the starting point $V_{\mathrm{SP}}$ and the battery impedance $Z_{i}$ into account.

$V_{\mathrm{OLO}}=V_{\mathrm{SP}}-Z_{i \mathrm{LIB}} \cdot I_{S}$

\subsubsection{B - Split current distribution}

An alternative current distribution and thus performance of the DBS occurs, if the initial voltage level prior applying the load current is below the OCV $\mathrm{MAX}$ level of the LAB. Figure 7 shows the split current distribution. Here, the LAB as well as the LIB are active from the start. The current will distribute in consideration of the the actual relationship between the internal battery resistances. As both batteries are active from the start, the calculation of the voltage drop can be done in consideration of the parallel impedance $Z_{\|}$calculated from the LIB's $\left(Z_{i}\right.$ LIB $)$ and the LAB's internal impedance $Z_{i}$ LAB .

$Z_{\|}=Z_{i \mathrm{LIB}} \| Z_{i \mathrm{LAB}}$

$V_{\text {Drop }}=Z_{\|} \cdot I_{S}$

Equation 10 shows the calculation of the on-load operational voltage of the DBS, taking the voltage at the starting point $V_{\mathrm{SP}}$ and the battery parallel impedance $Z_{\|}$into account.

$V_{\mathrm{OLO}}=V_{\mathrm{SP}}-Z_{\|} \cdot I_{S}$

This results in an increase of performance and a decrease of voltage drop.

\subsubsection{C-Combined distribution}

The third possibility of current split occurs under different conditions. This event takes place, if the initial voltage is again above the maximum OCV level. Here the load power gets so high, that the system voltage drops below the $\mathrm{OCV}_{\text {MAX }}$ level and the LAB gets activated. The qualitative presentation of this effect is shown in Fig. 8.

Again assumed, the consumer load requires the load current $I_{S}$ for faultless operation, the LIB alone delivers the

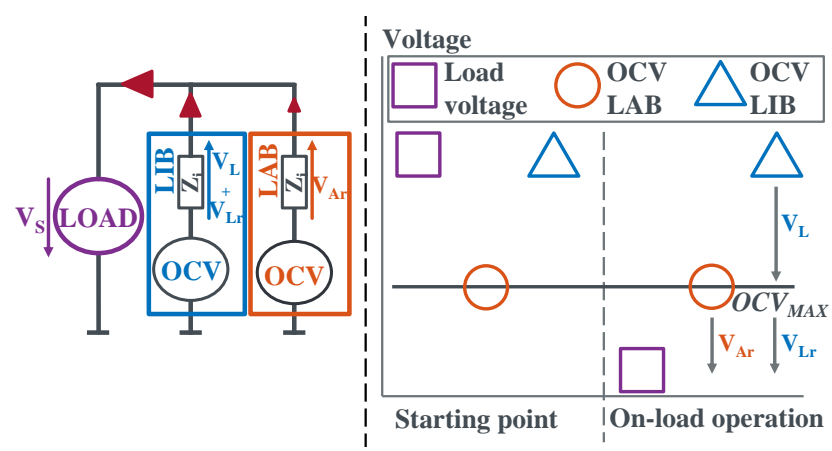

Figure 8. DBS Performance - Case C: Combined distribution.

threshold current $I_{\text {Thr }}$ until the voltage level drops due to the LIB's $Z_{i}$ LIB below the OCV $V_{\text {MAX }}$. The remaining current $I_{\mathrm{r}}$ required by the load is split up between the two batteries to $I_{\mathrm{Ar}}$ and $I_{\mathrm{Lr}}$. Thus, the voltage drops further, caused by the remaining current over the combined internal resistances $Z_{\|}$. The following equations detail the relationship.

$I_{\mathrm{Thr}}=\frac{\left(V_{\mathrm{SP}}-\mathrm{OCV}_{\mathrm{MAX}}\right)}{Z_{i \mathrm{LIB}}}$
$I_{\|}=I_{S}-I_{\mathrm{Thr}}$
$V_{\mathrm{OLO}}=\mathrm{OCV}_{\mathrm{MAX}}-\left(Z_{\|} \cdot I_{\|}\right)$

\subsection{Comparison of the predictive estimation methods}

To verify the functionality of the presented method for the predictive performance estimation for a DBS we perform the following test setup. We use a DBS as shown in Fig. 2 with a $70 \mathrm{Ah}$ LAB in an AGM design and as a high power battery a $10 \mathrm{Ah}$ LIB with a NMC/Carbon chemistry. Both batteries are connected next to each other with a $500 \mathrm{~mm}$ long and $50 \mathrm{~mm}^{2}$ sized copper wire, located in the vehicle's trunk. Prior applying load to the DBS, the batteries get constant voltage/constant current charged at different voltage levels from 13.0 to $14.5 \mathrm{~V}$. If the charging current decreases below a specified value, here $6 \mathrm{~A}$, the charging is stopped for a $10 \mathrm{~s}$ time period. After this, the DBS gets discharged with a $10 \mathrm{~s}$ current pulse at different intensities from 20 up to $500 \mathrm{~A}$. The test is performed at a controlled temperature of $25^{\circ} \mathrm{C}$. The voltage is recorded $2 \mathrm{~s}$ after starting the discharge pulse and displayed in Fig. 9 with a purple cross. For estimating the voltage drop, based on the presented method in Sect. 3.2, two parameters are presented: the initial system voltage $V_{\mathrm{SP}}$ and the applied current $I_{S}$, as these two are available in today's vehicles as well. This estimation method, also called hybrid estimation uses case-related one of the three presented ways for calculation and is represented by a red circle. To compare the newly established method with the wellknown approach, used for standalone battery systems, we perform this estimation as well. To analyze the performance we estimated the voltage drop on basis of the Case B - The split current approach, without any distinction of the initial voltage level. 


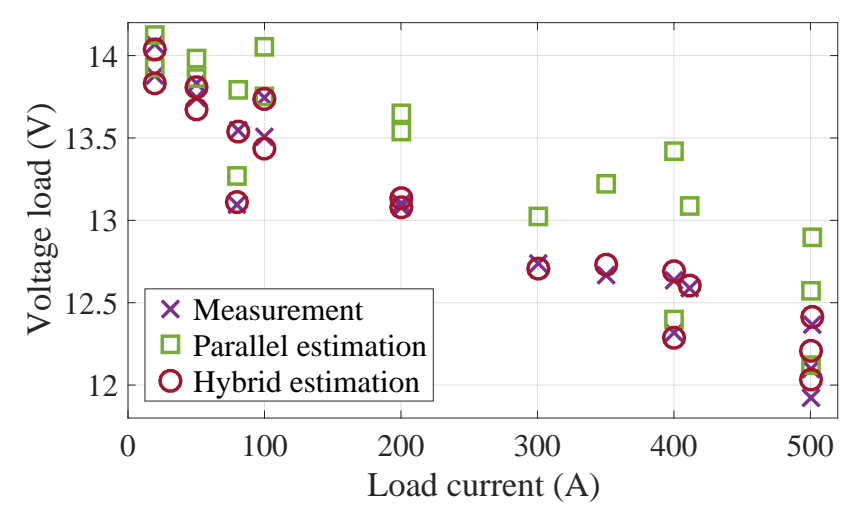

Figure 9. DBS Performance - Measurement and estimations at different starting voltages and pulse currents.

Table 1. Maximum and average errors of the presented estimation methods.

\begin{tabular}{lrr}
\hline Type of error & $\begin{array}{r}\text { Parallel } \\
\text { estimation }\end{array}$ & $\begin{array}{r}\text { Hybrid } \\
\text { estimation }\end{array}$ \\
\hline Maximum error (V) & 0.78 & 0.28 \\
Average error (V) & 0.32 & 0.04 \\
Maximum rel. error (\%) & 44 & 12 \\
Average rel. error (\%) & 25 & 5 \\
\hline
\end{tabular}

This one, called "Parallel estimation" is used for reference and marked with a green square in Fig. 9. The hybrid estimation yields to significant better results compared to the parallel estimation method. Especially in events with high starting voltages the parallel estimation results in too large variations, while the hybrid method is able to differentiate between the varying initial voltage levels. The algorithm is able to adapt itself depending on the present case. Table 1 gives a summary of the maximum and average errors obtained by the estimation methods in our test setup.

As presented in this article the estimation is done with simple battery models especially regarding the LAB. The usage of battery models of higher accuracy would achieve even better results.

\section{Conclusions}

The DBS is a new and powerful approach to meet the rising requirements for a stable $12 \mathrm{~V}$ power network of vehicles, due to high power consumers as well as autonomous driving functions. As this system has no ability to control the current distribution between the two batteries, the current share depends on the batteries' status. A variation in SOC and temperature results in a change of current share between the individual batteries and influences the DBS's performance. Additionally, the initial voltage before a pulse load shows a noticeable impact as the LIB has to take over more current at voltage levels above the LAB's OCV. Therefore, to predict the voltage drop and thus the performance of the DBS, the well-known method using an internal resistance based calculation is not applicable. The developed method in this article calculates the voltage drop in consideration of the present battery parameters as well as the initial voltage. Depending on the initial voltage and the overvoltage level of the LAB, the voltage drop is calculated by one of the three proposed methods. For lower consumer loads at high voltage levels only the LIB is used for estimation purposes. At low voltages in the range of the LAB's OCV the performance estimation is premised on the parallel connection of the two batteries' internal resistance and results in the least voltage drop. The remaining situations are determined by the hybrid method, combining case one and case two. Vehicle measurements show a very good estimation rate with a maximum deviation of $12 \%$ and an average deviation of $5 \%$. Compared to the internal resistance method with an maximum $44 \%$ and average deviation of $25 \%$. The proposed calculations are easy to handle, for a huge scattering of usable batteries and show a good computational efficiency. This enables to significantly extend the availability and duration of features such as start/stop or engine-off coasting. Future topics for research are the application of the presented method to other power network configurations and topologies with more than one energy storage. One example is the application to vehicle power networks with more than one voltage level e.g. a Mild-Hybrid Vehicle with a $12 \mathrm{~V}$ power network as well as a $48 \mathrm{~V}$ one. In this case the $12 / 48 \mathrm{~V}$ power converter would take the LIB's place and provide the power demand of high power applications until the voltage drops to a level where the LAB gets active.

Acknowledgements. This contribution was developed within the scope of the project Drive Battery 2015 (Intelligente Steuerungsund Verschaltungskonzepte für modulare Elektrofahrzeug- Batteriesysteme zur Steigerung der Effizienz und Sicherheit sowie zur Senkung der Systemkosten - AEV-Subproject: Optimierung des Energiemanagements von Fahrzeugen mit Lithium-Ionen Starter- und Bordnetzbatterien) which is funded by the BMWi (Bundesministerium für Wirtschaft und Energie) under the grant number 03 ET6003 I. The responsibility for this publication is held by the authors only.

Edited by: D. Killat

Reviewed by: two anonymous referees

\section{References}

Atkins, P., De-Paula, J., and Schleitzer, A.: Physikalische Chemie, Band 1, John Wiley \& Sons, New York, 2006.

Chen, M. and Rincon-Mora, G.: Accurate electrical battery model capable of predicting runtime and I-V performance, IEEE Transactions on Energy Conversion, 21, 504-511, 2006. 
Gehring, R., Froeschl, J., Kohler, T., and Herzog, H. G.: Modeling of the Automotive 14 V Power Net for Voltage Stability Analysis, IEEE Vehicle Power and Propulsion Conference, Dearborn, MI, 7-10 September 2009, 71-77, 2009.

Naumann, R.: Spanungs- Strom- und impdanzbasierte Methoden der Batteriediagnose: Möglichkeiten und Grenzen, HdT: Energiemanagement und Bordnetze, 11-32, 2004.

Netter, F., Xu, Z., and Gauterin, F.: Complexity Adaptation of Simulation Models in a Function-Based Modular Framework, IEEE 5th International Conference on Simulation and Applied Optimization (ICMSAO), Hammamet, Tunisia, 28-30 April 2013, 16, 2013.

Rizoug, N., Feld, G., Bouhali, O., and Mesbahi, T.: Micro-hybrid vehicle supplied by a multi-source storage system (battery and supercapacitors): Optimal power management, 7th IET International Conference on Power Electronics, Machines and Drives (PEMD 2014), Manchester, UK, 8-10 April 2014, 1-5, 2014.
Schindler, M.: Mehr-Batterie-System für Mikro-Hybrid-Fahrzeuge auf Basis von Blei-Sëure- und Lithium-Ionen-Technologie, IKA Verlag, 102-194, 2014.

Thele, M., Schiffer, J., Karden, E., Surewaard, E., and Sauer, D. U.: Modeling of the charge acceptance of lead-acid batteries, J. Power Sources, 168, 31-39, 2007.

Zhang, Z., Rick, A., and Sisk, B.: Model Development and Simulations of $12 \mathrm{~V}$ Dual Batteries towards Design Optimization of Microhybrid Vehicles, SAE Technical Paper, 1199, 2015. 